\title{
La recepción de Althusser en México: el caso Carlos Pereyra
}

Alfonso Vázquez

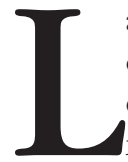

a influencia del pensamiento de Louis Althusser fue decisiva en América Latina durante las décadas de los años setenta y ochenta. En el caso de México, ese influjo se manifestó en la obra de filósofos como Adolfo Sánchez Vázquez y muchos de sus jóvenes discípulos como Cesáreo Morales, Carlos Pereyra, Mariflor Aguilar, Luis Salazar y otros más.

Cesáreo Morales, por ejemplo, en su ensayo "El althusserismo en México", publicado en 1984, en los números 13 y 14 de la revista Dialéctica, indica que fue precisamente Sánchez Vázquez el primero en realizar una recepción crítica de la obra de Louis Althusser y en mantener con ella un diálogo polémico que no acabó sino hasta 1978 con la publicación de la obra Ciencia y revolución (El marxismo de Althusser), pero yo mencionaría que ese diálogo se mantuvo constante e ininterrumpido hasta bien entrados los años noventa y quizá hasta la muerte del decano del marxismo en México, acaecida en 2011.

Morales refiere también que la recepción del althusserismo en México se puede clasificar en al menos tres etapas: una primera, de aparición y difusión, que va de 1965 a 1974, que abarca, entre otros eventos, la posesión de los primeros libros de Althusser en manos de Sánchez Vázquez y la traducción de Pour Marx por la editorial Siglo XXI bajo el título ya clásico de La revolución teórica de Marx en 1966; otra más, de planteamiento de problemas que comprende los años de 1975 a 1978, y que se caracteriza por el seguimiento del ritmo de la política y la proliferación de seminarios y centros de reflexión sobre la cultura; y una tercera, denominada por el propio Morales, aunque enfatizada por Gabriel Vargas Lozano en su Esbozo histórico de la filosofía en México (siglo XX), como "secularización del marxismo", ${ }^{1}$ que va de 1978

${ }^{1}$ Gabriel Vargas Lozano, Esbozo histórico de la filosofía en México (siglo XX) y otros ensayos. México, Consejo para la Cultura y las Artes de Nuevo León/UANL, Facultad de Filosofía y Letras, 2005, p. 109. 
a 1981, y que se caracterizó por "pensar el marxismo en otra forma, no como una religión sino como un pensamiento estricto", ${ }^{2}$ es decir, por el empleo de éste como una herramienta analítica y explicativa de la realidad social que, a su vez, abonó en el planteamiento de nuevas problemáticas teóricas que desembocaron en un profundo y riguroso estudio del Estado y la democracia, entre otros temas.

Así pues, el periodo que comprende la recepción y el desarrollo teórico del althusserismo en México mediante la asimilación, discusión, problematización y desarrollo de sus tesis abarca casi quince años: desde 1965 hasta 1981.

En esos años, como quizá en ningunos otros más de la filosofía en México, se desarrolló un pensamiento creativo que desde las coordenadas del marxismo crítico abrió el camino a nuevos campos problemáticos y nuevas formas de abordarlos, cuyas aportaciones y alcances aún están por ser evaluados, de acuerdo a lo que Gabriel Vargas Lozano indica en su libro ya mencionado.

Por otro lado, los acontecimientos políticos que en ese momento marcaron dicha etapa del pensamiento filosófico en nuestro país fueron fundamentalmente tres: 1) la movilización estudiantil de 1968 y su represión; 2) la reforma política de 1977 que legalizó la actividad del Partido Comunista Mexicano (PCM) y abrió los espacios para la participación política de muchas organizaciones que hasta ese momento realizaban su trabajo en la clandestinidad, y 3 ) la unificación de buena parte de las izquierdas en una organización partidaria de tendencia socialista que integró al propio PCM en el año de 1981: el Partido Socialista Unificado de México (PSUM).

Es decir, como bien indica el propio Morales en su ensayo ya citado, publicado posteriormente en 2007 como Louis Althusser (1918-1990) en su libro Fractales: pensadores del acontecimiento: "marginal o importante, el pensamiento de Althusser en México sólo encuentra su lugar en el interior de la producción intelectual que siguió al movimiento de 1968, enriquecida más tarde por el exilio latinoamericano". ${ }^{3}$

Aunque habría que explicar también cuáles fueron las causas que llevaron a una movilización política como la de 1968 y, sobre todo, especificar la dinámica propia de la producción intelectual en esos años, ya que muchas veces se suele pensar de manera errónea que el movimiento del 68 surgió casi por generación espontánea sin ningún tipo de determinaciones y sobredeterminaciones que lo configuraran.

Sobre lo primero, habría que mencionar que en ese momento se vivía en México una importante efervescencia política que no sólo fue decisiva para

${ }^{2}$ Cesáreo Morales, Fractales: pensadores del acontecimiento. México, Siglo XXI, 2007 , p. 79.

${ }^{3}$ Ibid., pp. 76-77. 
la gestación del movimiento estudiantil, sino que también es explicable por factores tanto internos como externos.

Entre los factores internos destaca el reflujo de las luchas populares y sindicales que sostuvieron los ferrocarrileros, los médicos y los maestros en los años cincuenta y sesenta, además de los procesos de oposición y resistencia a los cacicazgos regionales en estados como Morelos y Guerrero, a través de los liderazgos de Rubén Jaramillo, asesinado en 1962 durante el periodo de gobierno de Adolfo López Mateos, y de Genaro Vásquez y Lucio Cabañas, muertos respectivamente en 1972 y 1974 . Además, habría que sumar el contexto geopolítico de la Guerra fría y el triunfo de la Revolución cubana en 1959.

Todo ello impactó en el proceso de concientización política de buena parte de la población mexicana e impulsó el trabajo organizativo de los estudiantes que desembocó en la gran movilización de 1968. También hay que recordar que las principales demandas del movimiento eran, entre otras, la liberación de todos los presos políticos del país, particularmente de los dirigentes ferrocarrileros Demetrio Vallejo y Valentín Campa, así como la derogación del delito de "disolución social" que criminalizaba toda posición política socialista.

Esto es importante señalarlo, ya que plantear al movimiento estudiantil del 68 como un acontecimiento fundacional sin otras determinaciones que lo expliquen es abonar indirectamente a la idea de una supuesta pax priísta que se resquebraja casi de manera inexplicable en el año de la movilización estudiantil, sin tomar en cuenta que desde la década de los cuarenta por lo menos ya existían distintas expresiones de descontento social hacia el sistema político priísta que se desarrollaron en forma diferenciada y desigual a lo largo y ancho de la República, conformando diversas dinámicas de lucha y resistencia política que muchas veces se llevaron a cabo de manera simultánea. ${ }^{4}$

Ahora bien, en lo que concierne al ámbito de la producción intelectual de aquellos años, particularmente en lo que respecta al "campo filosófico", habría que valorar la importancia que tuvo la aparición de Filosofía de la praxis de Adolfo Sánchez Vázquez en el año de 1967. En lo particular, pienso que la publicación de esa obra, sumada a la experiencia política decisiva que dejó la movilización estudiantil en muchos universitarios y la lectura de los libros de Althusser por algunos dirigentes del movimiento recluidos en Lecumberri, explica en buena medida el proceso de evolución de una producción teórica formidable en el ámbito de la filosofía y el pensamiento social como quizá nunca antes se había dado en el horizonte de la cultura mexicana del siglo XX.

En lo que respecta a esto, el propio Morales señala, como ya se mencionó, que en el proceso de difusión del althusserismo fue fundamental la recepción

${ }^{4}$ Para tener un panorama más amplio de lo que aquí se expone, consúltese el libro de Fritz Glockner, Memoria roja. Historia de la guerrilla en México (1943-1968). México, Ediciones B, 2007. 
crítica que de ella hizo Adolfo Sánchez Vázquez, ya que fue el primero en tener en sus manos un ejemplar de Louis Althusser, además de ser también el primero en mencionarlo en un trabajo de investigación como fue su libro Filosofía de la praxis, que cuestionaba los principios de la interpretación althusseriana del marxismo, a la par que se deslindaba de todo indicio de humanismo teórico en su propia propuesta interpretativa.

En efecto, a lo largo de la obra de Adolfo Sánchez Vázquez se manifiesta una recurrente referencia al pensamiento de Althusser, ya sea para analizar y criticar sus tesis o bien para contrastar su interpretación del marxismo basada en la categoría de la praxis y en los Manuscritos económico-filosóficos de 1844 con la concepción cientificista y anti-humanista del filósofo francés.

De cualquier modo, como también indica el propio Cesáreo Morales en otro trabajo titulado "La investigación filosófica en la UNA M: los años ochenta", es evidente cómo Sánchez Vázquez en Ciencia y revolución, "con paciencia y hasta con una simpatía intelectual inocultable, emprende una exposición crítica del althusserismo: desde su estructura conceptual misma, como se debe; clarificando sus axiomas y teoremas y mostrando sus naturales insuficiencias". 5 Quizá con ello, Sánchez Vázquez le daba la razón a Althusser cuando indicaba que "toda filosofía está como habitada por su antagonista, por el espectro de su contrario". ${ }^{6}$

Del magisterio de Adolfo Sánchez Vázquez, como ya se señaló al principio de este trabajo, se formaron importantes personalidades teóricas e intelectuales que bajo su influjo, pero también bajo el de las principales corrientes del marxismo teórico de la época, como el althusserismo, desarrollaron una obra significativa que removió las inercias en ese pensamiento que se concebía muchas veces como clausurado y definitivo.

Justo es el caso de Carlos Pereyra, un brillante discípulo de Adolfo Sánchez Vázquez con quien, a pesar de su cercanía, igualmente no dejó de polemizar y sostener posiciones críticas a lo largo de su vida.

Carlos Pereyra ingresa a la Universidad en el año de 1959, justo el año del triunfo de la Revolución cubana, y vive como estudiante el movimiento de 1968, a la par que milita en las juventudes del PCM y posteriormente en la Liga Comunista Espartaco.

Además, en los años setenta se vincula orgánicamente con la Tendencia Democrática del Sindicato Único de Trabajadores Electricistas de la República Mexicana (SUTERM), encabezada por Rafael Galván, y milita en el sindicalismo universitario. "Posteriormente -indica Arnaldo Córdova- fue miembro fun-

${ }^{5}$ C. Morales, "La investigación filosófica en la UNAM: los años ochenta", en Omnia. Revista de la Coordinación General de Estudios de Posgrado. México, unAM, diciembre, 1987, núm. 9. en http://www.posgrado.unam.mx/es/omnia-num-9.

${ }^{6}$ Emilio de Ípola, Althusser, el infinito adiós. Buenos Aires, Siglo XXI, 2007, p. 166. 
dador y dirigente del Movimiento de Acción Popular; fue miembro fundador del Partido Socialista Unificado de México, a cuyo Comité Central perteneció después de su último Congreso, y fue miembro fundador del Partido Mexicano Socialista". ${ }^{7}$ Muere el 6 de junio de 1988, un mes antes de llevarse a cabo las emblemáticas elecciones presidenciales de aquel año en donde por primera vez la izquierda política a través de un amplio bloque de fuerzas históricas agrupado en el Frente Democrático Nacional, se encontró en posibilidades reales de arrebatarle el poder gubernamental al PRI.

Es decir, nadie quizá como Pereyra puede ilustrar con su biografía la trayectoria de las distintas etapas de lucha política de la izquierda mexicana en el último cuarto del siglo Xx. Simultáneamente a su actividad política, desarrolló una importante obra teórica que no puede concebirse sin la realidad histórica de la que surge ni de las consecuencias prácticas que se le derivan.

Por esa razón, acaso el pensamiento de Carlos Pereyra sea uno de los más emblemáticos de la recepción del althusserismo en México, en primer lugar, porque con su investigación teórica contribuyó a esclarecer conceptos fundamentales que tuvieron una repercusión en el plano ideológico y en la lucha política de las izquierdas de los años setenta y ochenta en nuestro país. En segundo lugar, porque la asimilación del pensamiento de Althusser nunca fue dogmática ni exenta de crítica, sino que supuso una revisión problemática de cada una de sus tesis y un riguroso proceso de análisis de cada uno de sus conceptos.

En ese sentido, el trabajo teórico de Carlos Pereyra es uno de los más originales no sólo dentro de las coordenadas del marxismo crítico, y del althusserismo en particular, sino también dentro del panorama de la filosofía contemporánea en México. Uno de los méritos de Pereyra es abandonar al marxismo como un sistema de reiteraciones y en cambio asumirlo como un sistema explicativo que sólo puede desarrollarse a condición de problematizar sus propios supuestos teóricos.

De ese modo, en uno de los ensayos de Configuraciones: teoría e historia, quizá el libro en donde se manifiesta de una manera más marcada el influjo del pensamiento de Louis Althusser en su obra, plantea que "el marxismo presenta la paradoja de ser la única teoría capaz de dar cuenta del movimiento de la totalidad social e intervenir en su curso y a la vez prolongar su estado de inacabamiento". ${ }^{8}$ Desde luego que esta premisa era también aplicable al propio marxismo de Louis Althusser.

${ }^{7}$ Adolfo Sánchez Vázquez et al., En memoria de Carlos Pereyra. México, unam, Facultad de Filosofía y Letras, 1989, p. 12.

${ }^{8}$ Carlos Pereyra, Filosofía, historia y política. Ensayos filosóficos (1974-1988). México, FCE/UNAM, 2010, p. 95. Cabe aclarar que en esta edición se compilan, por Gustavo Ortiz Millán y Corina Yturbe, los tres libros que Pereyra publicó en vida. Se trata 
Aquí conviene hacer una aclaración: si bien es cierto que el pensamiento de Louis Althusser es decisivo en la obra de Carlos Pereyra, sobre todo en la primera etapa de su desarrollo, también hay que decir que éste no es su única influencia: desde sus primeras obras aparecen ya los nombres de marxistas críticos como Lukács, Kosík, Gramsci y otros más. Además, no debemos dejar de mencionar que el magisterio y la obra de Adolfo Sánchez Vázquez fue igual de decisiva para que asumiera una forma distinta de asimilar el marxismo en México, elevando el nivel de análisis y propiciando un rigor teórico que no se había llevado a cabo antes con tal claridad y precisión, todo ello sin olvidar la vinculación orgánica con la práctica política.

Desde esta perspectiva, en su primer libro Política y violencia, publicado en 1974 por el Fondo de Cultura Económica en la colección Testimonios del Fondo, Pereyra realiza una puntual y rigurosa reflexión en torno a la violencia desde el enfoque de la teoría política. Para Pereyra no se puede hablar del papel de la violencia en la política sin especificar antes la función del Estado.

En ese sentido, siguiendo el pensamiento de Louis Althusser y Nicos Poulantzas, entre otros, plantea que el Estado no debe pensarse como un mero aparato concebido explícitamente para el dominio de una clase social en particular, sino más bien debe plantearse como una estructura que al expresar la correlación de fuerzas y las tensiones existentes entre los grupos y clases que conforman una sociedad determinada, tendrá como principal función, más allá de las tareas propias de la administración pública, mantener y reproducir el orden social vigente, y no tanto mantener y reproducir la dominación de una clase social específica.

Es decir, si las clases sociales dominantes mantienen el control político del aparato del Estado, esto se debe fundamentalmente a que el Estado posibilita tal estructura de dominación en la medida en que es expresión de las relaciones sociales existentes, y no porque haya sido hecho a modo para una clase social en particular; de ahí que si esa estructura de las relaciones sociales cambia, también se modificará necesariamente la configuración del propio Estado.

De tal forma que siguiendo el pensamiento de Louis Althusser señala que el Estado para conseguir su principal objetivo, que es el de mantener y reproducir las relaciones sociales existentes, distribuye su función en dos grandes aparatos que son los aparatos represivos e ideológicos del Estado.

Por aparatos represivos, Pereyra concibe todas aquellas instituciones en donde se organiza y administra la violencia física y que son fundamentalmente la policía, el ejército y las prisiones. Mientras que por aparatos ideológicos refiere a todas aquellas instancias encargadas de producir "un efecto ilusorio 
de desconocimiento del sitio que ocupan los dominados en las relaciones sociales establecidas" 9 y de difundir cierto tipo de ideas para mantener el control político de la población, aunque no estén necesariamente vinculadas con el aparato estatal y conserven una relativa autonomía, y entre las que se encuentran los centros educativos, las Iglesias, las universidades, los sindicatos y los medios de comunicación, entre otras.

Cabe mencionar que su concepción del Estado, en tanto instancia que distribuye su función en esos dos aparatos, se modificará en cuanto incorpora a su pensamiento las aportaciones teóricas de Antonio Gramsci, según las cuales las instituciones del Estado son aquellas en donde se ejerce y distribuye el poder político como los órganos de gobierno, las magistraturas, el parlamento o el ejército, mientras que las instituciones de la sociedad civil serían instancias de articulación de los intereses privados de los distintos grupos que conforman a ésta como los sindicatos, las universidades o los organismos empresariales, aunque la idea de una concepción no instrumentalista y reduccionista del Estado como estructura benéfica de una clase social específica prevalezca.

De cualquier forma, en Política y violencia, la relación entre ambos aparatos no es una relación de articulación simple, sino una relación de articulación compleja y complementaria, es decir, uno no actúa cuando el otro deja de funcionar, sino que la acción de ambos se desarrolla simultáneamente, ya que la ideología para Pereyra no es más que "la continuación de la violencia por otros medios", ${ }^{10}$ aunque nunca aparece de manera aislada.

En ese sentido, otra de las sugerentes y lúcidas aportaciones que Pereyra establece en este libro fundamental es aquella que refiere a la relación entre legitimidad y violencia. Para Pereyra la legitimidad no es la esencia de un gobierno, sino el poder. Y el poder no puede concebirse sin el papel de la violencia en su estructuración, de tal modo que cuando se pierde legitimidad en un gobierno determinado no se está perdiendo sólo el reconocimiento o la aprobación de un sector significativo de la población, sino se está perdiendo fundamentalmente poder.

Por tal razón, cuando se pierde legitimidad, el gobierno que la pierde tiende a usar en mayor medida los aparatos represivos del Estado, es decir, incrementa el uso de la violencia política, y cuando un gobierno tiene una legitimidad alta, el uso de la violencia disminuye. De tal modo, para Pereyra, a mayor legitimidad menor violencia, y a menor legitimidad el uso de la violencia es mayor.

Otra tesis que establece, tomando como referente el pensamiento de Lukács, y que está relacionada con la anterior, es aquella que señala la relación existente entre conciencia fáctica y conciencia atribuida, planteando que la

\footnotetext{
${ }^{9}$ Ibid., p. 35.

${ }^{10}$ Ibid., p. 40.
} 
primera es aquella conciencia que en los hechos es asumida por una clase social específica, mientras que la segunda no se refiere a la conciencia que una clase debería asumir, es decir, no es una categoría con una pretensión normativa, sino que alude a la correspondencia entre esa conciencia y el lugar o el papel que la clase social desempeña en el proceso de producción.

De tal modo que, para Pereyra, a menor distancia entre conciencia atribuida y conciencia fáctica, el uso de la violencia política de carácter represivo es mayor, ya que la conciencia que se le atribuye a las clases dominadas deja de ser un mero supuesto teórico y se convierte en una realidad efectiva, es decir, esas clases actúan conforme al lugar que ocupan en el proceso productivo y, por lo tanto, delinean con firmeza una estrategia destinada a romper con las relaciones de dominación; mientras que cuando sucede lo contrario, es decir, cuando es mayor la distancia entre conciencia atribuida y conciencia fáctica, el uso de la violencia política de carácter represivo es menor o nulo.

Otra de las aportaciones fundamentales de Pereyra es su análisis en torno al concepto de ideología que desarrolla en el libro Configuraciones: teoría e historia, publicado en 1979. Para Pereyra, el término ideología se caracteriza por su ambigüedad y por su multivocidad, es decir, por ser una palabra que es usada "como vehículo de dos conceptos diferentes pertenecientes a marcos teóricos distintos". ${ }^{11}$ Por tal motivo lo más conveniente es "distinguir con precisión su significado en cada caso y evitar la ambigüedad por la vía de una rigurosa determinación contextual, es decir, dejando que el tipo de discurso decida en cada caso de qué concepto se trata". ${ }^{12}$

De esta manera, lo primero que establece Pereyra es que estos dos conceptos a través de los cuales puede ser usado el término ideología son el concepto epistemológico y el concepto sociológico. El concepto epistemológico de ideología refiere al conjunto de ideas, representaciones y creencias que no se encuentran justificadas teóricamente, y que son expresadas a través de enunciados que no están debidamente sustentados en razones suficientes; concebidas las primeras y formulados los segundos por razones extrateóricas, es decir, por razones ajenas al plano del conocimiento: razones políticas, económicas, de clase, raciales, nacionales, etcétera.

De tal modo que si se califica a un discurso de ideológico, desde esta perspectiva, no sólo se deben mostrar las razones por las cuales se encuentra insuficientemente sustentado, sino también las causas por las que ese discurso se impone como verdadero a través de la función social que cumple.

Por otro lado, el concepto sociológico del término ideología se refiere a la manera en que un discurso es capaz de articular los intereses o aspiraciones

${ }^{11}$ Ibid., p. 83.

${ }^{12}$ Idem. 
de una clase o grupo social de acuerdo al lugar que ocupan en el conjunto de las relaciones de producción; de esta manera, el concepto sociológico de ideología se usa para describir "la relación existente entre un discurso, sin importar la verdad o falsedad del mismo, y sus usuarios (receptores o emisores), en virtud de los propósitos y aspiraciones de una clase o grupo social históricamente condicionados por el lugar que esa clase o grupo ocupa con respecto al poder y al conjunto de las relaciones sociales". ${ }^{13}$

Así pues, la teoría de la ideología en Pereyra va más allá de lo que plantea el propio Althusser, puesto que describe una necesaria relación entre la producción de conocimientos y el plano social en el que esa producción se lleva a cabo. Además, la ideología en ninguno de los dos conceptos a los que se refiere, de acuerdo con Pereyra, se presenta sólo como una representación falsa de la realidad, sino que, ya sea desde el concepto epistemológico o sociológico, la ideología siempre va a ser algo más que una concepción errónea de la realidad, ya que en el primer caso se asume como verdadera por la función social que cumple -independientemente de la insuficiencia de las supuestas razones en las que se encuentra sustentada-; y en el segundo, la ideología, al referirse al discurso a través del cual las aspiraciones y anhelos de ciertas clases o grupos sociales se articulan con objetivos políticos precisos, se coloca más allá de lo verdadero o de lo falso.

De tal modo, que hablar de la ideología burguesa como falsa y de la ideología proletaria como verdadera carece de toda pertinencia, puesto que son sólo discursos que articulan los intereses de esas clases en función del lugar que ocupan en el proceso de producción, y por esa razón no son ni buenas ni malas, ni mucho menos verdaderas o falsas; al caso serán eficaces o adecuadas en función del éxito o fracaso de sus objetivos.

Así pues, como indica Adolfo Sánchez Vázquez, Pereyra "al introducir sus dos conceptos -epistemológico y sociológico- de ideología puede superar el foso abierto por Althusser entre ideología y ciencia", ${ }^{14}$ ya que para Pereyra el conocimiento al ser un producto social, presenta necesariamente una dimensión ideológica en la medida en que puede o no ser producido o apropiado por distintos grupos o clases que conforman la estructura social.

O sea, "pertrechado con estos dos conceptos de ideología -que no entrañan dos tipos de ideología- Pereyra logra desenredar algunas de las cuestiones en las que Althusser se había enredado". ${ }^{15}$

También convendría hablar de la reivindicación que hace Pereyra de conceptos althusserianos como "sobredeterminación" y "causalidad estructural"

${ }^{13}$ Ibid., p. 85.

${ }^{14}$ A. Sánchez Vázquez et al., op. cit., p. 16.

${ }^{15}$ Idem. 
que le permiten desarrollar una explicación de la historia no basada en premisas reduccionistas como el economicismo o cualquiera de sus derivaciones, y que le llevan a continuar problematizando la tesis althusseriana de que la historia es un proceso sin sujeto.

Todo este desarrollo teórico lo encontramos en la primera etapa de su pensamiento, particularmente en los libros Política y violencia (1974) y Configuraciones: teoría e historia (1979), y todavía se hallan sus resonancias en el libro de madurez El sujeto de la historia (1984), aunque en éste la influencia del pensamiento de Antonio Gramsci comienza a tener ya una importancia decisiva.

Posteriormente, con la asimilación y la recuperación crítica de los clásicos de la filosofía política, particularmente de los teóricos de la democracia como Toqueville, Macpherson, Rawls, Apel o Habermas, entre otros, Pereyra planteará la necesidad de pensar el socialismo en clave democrática, ya que no podía concebirlo sin la vinculación con un proceso político en donde las masas participaran abiertamente en la toma de decisiones.

Este interés por conceptualizar la democracia y por comprender la naturaleza y función específica del Estado, es decir, por enriquecer el marco teórico de un campo problemático tan fundamental como es el de la filosofía política, no provenía de una actitud meramente académica, sino que se enmarcaba en el primer plano de los problemas que le planteaba su vinculación con la práctica política misma.

Si como dice Cesáreo Morales, "se consumó el ciclo del althusserismo"16 en México, entonces, los problemas que propició, las reflexiones que sugirió y, sobre todo, la estela de pensadores que como Carlos Pereyra de una u otra forma abarcó, mantendrán su vigencia en la medida en que remuevan "las incertidumbres y certidumbres de cada día". ${ }^{17}$

En ese sentido, y sólo en ese sentido, seremos entonces "post-althusserianos", es decir, herederos de una tradición de pensamiento que nos permita seguir problematizando el presente y planteando nuevas alternativas ante sus limitaciones. Tal como fue el caso de la intervención teórica de Carlos Pereyra en nuestro país, y que ahora es un legado del que podemos valernos para seguir pensando con rigor los problemas de nuestro nuevo tiempo mexicano.

${ }^{16}$ C. Morales, Fractales: pensadores del acontecimiento, p. 87.

${ }^{17}$ Idem. 